\title{
Targeting HDACs: A Promising Therapy for Alzheimer's Disease
}

\author{
Ke Xu, ${ }^{1}$ Xue-Ling Dai, ${ }^{2,3}$ Han-Chang Huang, ${ }^{2}$ and Zhao-Feng Jiang ${ }^{2,4}$ \\ ${ }^{1}$ College of Life Science, Capital Normal University, Beijing 100048, China \\ ${ }^{2}$ College of Arts and Science, Beijing Union University, Beijing 100191, China \\ ${ }^{3}$ Department of Physiology and Pathophysiology, Peking University School of Basic Medical Sciences, Beijing 100191, China \\ ${ }^{4}$ Beijing Key Laboratory of Bioactive Substances and Functional Foods, Beijing Union University, Beijing 100191, China
}

Correspondence should be addressed to Zhao-Feng Jiang, zhaofeng@buu.edu.cn

Received 16 May 2011; Revised 22 July 2011; Accepted 23 July 2011

Academic Editor: Florian Lang

Copyright () $2011 \mathrm{Ke} \mathrm{Xu}$ et al. This is an open access article distributed under the Creative Commons Attribution License, which permits unrestricted use, distribution, and reproduction in any medium, provided the original work is properly cited.

\begin{abstract}
Epigenetic modifications like DNA methylation and histone acetylation play an important role in a wide range of brain disorders. Histone deacetylases (HDACs) regulate the homeostasis of histone acetylation. Histone deacetylase inhibitors, which initially were used as anticancer drugs, are recently suggested to act as neuroprotectors by enhancing synaptic plasticity and learning and memory in a wide range of neurodegenerative and psychiatric disorders, such as Alzheimer's disease (AD) and Parkinson's disease (PD). To reveal the physiological roles of HDACs may provide us with a new perspective to understand the mechanism of AD and to develop selective HDAC inhibitors. This paper focuses on the recent research progresses of HDAC proteins and their inhibitors on the roles of the treatment for $\mathrm{AD}$.
\end{abstract}

\section{Introduction}

Alzheimer's disease (AD), including familiar AD and sporadic $\mathrm{AD}$, is a progressive neurodegenerative disease and the most common form of dementia. This late-onset disorder is characterized by memory loss and cognitive impairment. The pathological features are neurofibrillary tangles (NFTs), insoluble $\beta$-amyloid $(\mathrm{A} \beta)$ plaques, and neuron loss [1]. According to the most convincing hypothesis at present, the "amyloid cascade hypothesis," it is believed that the accumulated $\mathrm{A} \beta$ peptide leads to a complex cascade of neuronal apoptosis and results in the pathogenesis of $\mathrm{AD}[2,3]$. Increasing evidence supports the notion that some genetic changes in familiar $\mathrm{AD}$, such as amyloid $\beta$ precursor protein (APP), presenile 1, 2 (PS1\&2), and apolipoprotein E, are linked to the overproduction of $A \beta$ [4]. Although we have known a lot about both familiar $\mathrm{AD}$ and sporadic $\mathrm{AD}$, it is still a long way to fully understand the pathogenesis of the diseases, especially sporadic AD. Epigenetics is a branch of genetics. Epigenetics studies the gene expression when the nucleotide sequences of a gene do not change, but rather other modification factors including histone acetylation and DNA methylation [5]. The epigenetic regulation offers a new way to understand $\mathrm{AD}$, especially sporadic $\mathrm{AD}$. $\mathrm{DNA}$ methylation has been previously proved to play a role in $\mathrm{AD}$, and recently several studies suggested that histone acetylation is involved in the etiology of $\mathrm{AD}[6,7]$. Histone acetylation and deacetylation are catalytic by histone acetyltransferases (HATs) and histone deacetylases (HDACs), respectively. The level of histone acetylation plays an important role in regulating the chromatin condensation and gene transcription [8]. HDACs regulate the level of histone acetylation and further affect some downstream gene expression. Abnormal acetylation of histone is involved in the pathology of $\mathrm{AD}$. HDAC proteins may be therapeutical targets to treatment for $\mathrm{AD}$. HDAC inhibitors have been reported to improve the memory and cognition in the mouse model of AD. HDAC inhibitors may be alternative drugs to potentially protect the impairment of cognition in AD patients. However, HDAC proteins serve a very distinct function in the brain. Therefore, the use of pan-HDAC inhibitors (nonselective HDAC inhibitors) in the treatment of neuropsychiatric disorders should be careful. To identify which numbers of HDAC family are involved in memory and learning is helpful in discovering the pathological mechanism of $\mathrm{AD}$ and in developing selective HDAC inhibitors. 


\section{Histone Deacetylase and Histone Deacetylase Inhibition}

2.1. Histone Deacetylase. HDAC proteins belong to an ancient protein family in many species. In fact, contrary to what is suggested by their names, certain HDACs mainly interact with nonhistone protein. In mammals, there are eighteen HDAC enzymes (Table 1), which are divided into four classes based on their homology to yeast: class I, class II, and class IV. Among these classes, class I, class II, and class IV are zincdependent enzymes, whereas class III is dependent on nicotinamide adenine dinucleotide $\left(\mathrm{NAD}^{+}\right)[9,10]$.

Class I of HDACs, which consists of HDAC 1, 2, 3, and 8, primarily localizes in the nucleus where they regulate histone acetylation. Class II of HDACs is divided into two subtypes: class IIa and IIb. Class IIa includes HDAC 4, 5, 7, and 9 and class IIb includes HDAC 6 and 10. Class IIa of HDACs shuttles between the nucleus and the cytoplasm in response to certain cellular signals, whereas class IIb of HDACs mainly locates in cytoplasm. In general, class III of HDAC is referred to as "sirtuins." Class III of HDACs includes SirT1-7, which share homologous sequence with the yeast Sir2 protein; SIRT 3,4 , and 5 are mitochondrial proteins [11]. Class IV HDAC is known to have only one member, HDAC11, which contains a catalytic domain located at the N-terminus. HDAC11 seems to be closely related to HDAC3 and HDAC8 [12], and it also mainly locates in the cellular nucleus [13].

2.2. Histone Deacetylase Inhibition and Alzheimer's Disease. In recent years, a variety of HDAC inhibitors have been developed (Table 1). The pan-HDAC inhibitors widely used in clinical research are valproic acid, trichostatin A, sodium 4phenylbutyrate, and vorinostat; these inhibitors interact with zinc-dependent HDAC protein (class I, class II, and class IV). Nicotinamide, as the precursor of $\mathrm{NAD}^{+}$, inhibits class III HDAC proteins [14].

Valproic acid (VPA), as well as lithium, inhibits $\mathrm{A} \beta$ peptide production in HEK293 cell transfected with Swedish $\mathrm{APP}_{751}$ [15]. VPA also significantly reduces $\mathrm{A} \beta$ plaque in $\mathrm{AD}$ transgenic mice. VPA decreases $\mathrm{A} \beta$ production by inhibiting GSK- $3 \beta$-mediated $\gamma$-secretase cleavage of APP and alleviates the memory deficits in AD mouse model [16]. Ricobaraza et al. suggested that 4-phenylbutyrate (PBA) decreases the phosphorylation of tau based on an increase of an inactive form of the GSK-3 $\beta$ in Tg2576 mouse model of AD [17]. PBA reinstates memory in both young and old Tg2576 mice and reverses learning deficits through clearance of intraneuronal $\mathrm{A} \beta$ accumulation and mitigation of endoplasmic reticulum (ER) stress [18]. Nicotinamide, a competitive inhibitor of class III $\mathrm{NAD}^{+}$-dependent HDACs, restores cognitive deficits in 3xTg-AD mice. Nicotinamide selectively reduces phosphorylation of tau at Thr231 site and increases the acetylated $\alpha$-tubulin [14]. Ding et al. found that tubacintreated HEK cells transfected with tau significantly attenuates tau phosphorylation at T231 [19]. However, the untoward effects of pan-HDAC inhibitors limit their application to clinic $[20,21]$. Tubacin and suramin are the most studied selective HDAC inhibitors; tubacin shows high selectivity for
HDAC6 [22], and suramin inhibits the activity of $\mathrm{NAD}^{+}$dependent class III SirT1 and SirT2 [23]. Here are the isoforms of HDACs, and the mainly used HDAC inhibitors are listed in Table 1.

Above facts indicate that some HDAC proteins may have a close relationship with those key proteins which are involved in AD. HDAC inhibitors may be used to treat AD by regulating the activity of HDAC proteins and phosphorylation of Tau. However, the mechanism of HDAC-regulated cellular signaling in $\mathrm{AD}$ pathology is needed to further investigate.

\section{Acetylation of Histone and Alzheimer's Disease}

Histone acetylation is involved in the gene expression through chromatin modification, and the acetylation was mainly in the N-terminal of histone $\mathrm{H} 3$ and H4. The cognitive deficit is one of the major clinical characteristics of $\mathrm{AD}$ patients. In mice, deregulation of histone acetylation is associated with age-dependent memory impairment. Aged mice display a specific deregulation of histone $\mathrm{H} 4$ lysine 12 (H4K12) acetylation and fail to initiate a hippocampal gene expression which is involved in memory consolidation, and vorinostat-treated mice display significant increase of $\mathrm{H} 4 \mathrm{~K} 12$ acetylation and restore learning-induced gene expression [24]. More and more evidence suggests that dysregulation of histone $\mathrm{H} 4$ acetylation is involved in AD pathology. Kilgore et al. reported that there is no difference in $\mathrm{H} 3$ or $\mathrm{H} 4$ acetylation in the hippocampus area between 6-month-old APP/PS1 mice and wild-type mice, but a significant reduction was observed in $\mathrm{H} 4$ acetylation in old APP/PS1 mice. The class I HDAC inhibitors sodium valproate, sodium butyrate, or vorinostat elevate histone $\mathrm{H} 4$ acetylation and restore contextual memory in a mouse model of $\mathrm{AD}$ [25]. Similarly, Francis et al. found that there is no difference in the acetylation level of histone $\mathrm{H} 4$ between the 4-monthold wild-type and the APP/PS1 mouse. After contextual fearconditioning training, the APP/PS1 mice display a reduced endogenous level of histone $\mathrm{H} 4$ acetylation, and the mice treated with trichostatin A (TSA), a class I and II HDAC inhibitor, had improved acetylated histone $\mathrm{H} 4$ level and were displayed hippocampal CA3-CA1 long-term potentiation (LTP) [26]. Ricobaraza et al. reported that the acetylation of histone H4 is decreased in the 16-month-old Tg2576 mouse brain compared with nontransgenic controls, while there is no difference in $\mathrm{H} 3$ acetylation. Phenylbutyrate, a class IIa HDAC inhibitor, ameliorates the cognitive deficits in Tg2576 mice and increases the neuronal acetylation of $\mathrm{H} 4$ and the expression of synaptic plasticity markers including GluR1, PSD95, and MAP2 [17]. What is more, in Tg2576 mouse model of $\mathrm{AD}$, the phenylbutyrate restores the dendritic spine density of hippocampal CA1 pyramidal neurons and significantly increases the expression of plasticity-related proteins like the NMDA receptor subunit NR2B and the synaptic scaffold SAP102 [18]. Taken together, histone acetylation H4 is involved in the pathology of $\mathrm{AD}$, and HDAC inhibitors may 
TABLE 1: HDAC isoforms and main used of pan-HDAC inhibitors.

\begin{tabular}{lccc}
\hline Histone subtype & Protein & Localization & Main HDAC inhibitors \\
\hline Class I (Zn++-dependent) & HDAC1, 2, 3, and 8 & Mainly nucleus & Valproic acid, butyrate, vorinostat, \\
trichostatin A, RGFP136 (HDAC3) & Trichostatin A, phenylbutyrate \\
Class IIa (Zn++-dependent) & HDAC4, 5, 7, and 9 & Nucleus/cytoplasm & Tubacin (HDAC6), trichostatin A \\
Class IIb (Zn++-dependent) & HDAC6 and 10 & Mainly cytoplasm & Nicotinamide, suramin (SirT1 and SirT2), \\
ClassIII (NAD+-dependent) & SirT1, 2, 3, 4, 5, 6, and 7 & Nucleus/cytoplasm/Mitochondria & \\
Class IV (Zn++-dependent) & HDAC11 & Mainly nucleus & \\
\hline
\end{tabular}

alter some important gene expression through regulating the histone acetylation.

\section{Histone Deacetylases and Alzheimer's Disease}

HDACs influence the level of histone acetylation, and HDAC inhibitors upregulate histone acetylation level and improve memory and learning. The HDAC inhibitors affect the activities of the proteins that play an important role in $\mathrm{AD}$, like $\mathrm{A} \beta, \mathrm{GSK}-3 \beta$, and tau protein. GSK-3 $\beta$, as a main tau phosphokinase, is linked to several mechanisms involved in $\mathrm{AD}$. Increasing evidence suggests that $\mathrm{A} \beta$ induces hyperphosphorylation of tau though the activation of GSK-3 $\beta$ [27, 28 ]. Therefore, inhibition of $\mathrm{A} \beta$-induced deficits of histone acetylation and hyperphosphorylation of tau is helpful to treat AD. HDAC inhibitors downregulate HDAC activity and thus increase the acetylation level of histone. At present, however, most available HDAC inhibitors are nonselective for HDACs because it is not fully understood which subtypes of HDACs have effects on development of AD. Identifying which subtypes of HDAC family members are involved in the pathology of $\mathrm{AD}$ is needed to further investigate, specifically, impairment of memory and learning.

4.1. Class I HDAC. Mice overexpressing HDAC2, but not HDAC1, result in decreased synaptic plasticity, synapse number and memory formation, and vorinostat could rescue the synaptic number and learning impairments in HDAC2overexpressing mice. Generally, HDAC2 negatively regulates learning and memory [35]. Akhtar et al. demonstrated that in mature neurons the upregulated level of HDAC2 affects the basic excitatory neurotransmission, implying that HDAC2 may play a role in synaptic plasticity [36].

McQuown et al. found that HDAC3-Flox-modified mice (deletion of HDAC3 in the hippocampus of CA1 area) or the mice treated with RGFP136 (selective inhibitor of HDAC3) increase histone acetylation and significantly enhance longterm memory, and the expression of the genes of nuclear receptor subfamily 4 , group $\mathrm{A}$, member $2(\mathrm{Nr} 4 \mathrm{a} 2)$ and $\mathrm{c}$ Fos is implicated in long-term memory [37]. Besides, Bardai and d'Mello suggested that HDAC3 is a protein with strong neurotoxic activity, and the toxic effect is cellular selective. HDAC3 is directly phosphorylated by GSK-3 $\beta$, and inhibition of GSK-3 $\beta$ protects against HDAC3-induced neurotoxicity [38].
4.2. Class II HDAC. HDAC6, as a cytosolic enzyme, catalyzes several nonhistone proteins, such as tubulin and HSP90 deacetylase [39, 40]. HDAC6 protein level in AD brains is significantly increased in cortex and hippocampus compared with the normal brains. Tubacin (a selective inhibitor of HDAC6) attenuates site-specific phosphorylation of tau, suggesting that HDAC6 plays a role in the AD [19]. The selective inhibition of HDAC6 dramatically enhances mitochondrial movement in hippocampal neurons. GSK-3 $\beta$ may be involved in the active regulation of HDAC6 by phosphorylation pathway. Therefore, abnormal mitochondrial transportation could be resulted from the misregulation of HDAC6 by GSK-3 $\beta$ [41]. Besides, oxidative stress is the main pathological feature of $\mathrm{AD}$, and selective inhibition of HDAC6 protects against oxidative-stress-induced neurodegeneration and promotes neurite outgrowth in cortical neurons [42].

HDAC4 is mainly localized in the cytoplasm of brain tissue, and abnormal expression of nuclear-localized HDAC4 promotes neuronal apoptosis while inactivation of HDAC4 suppresses neuronal cell death [43]. Therefore, HDAC4 may play an important role in nerve function.

4.3. Class III HDAC. Gao et al. found a significant reduction of SirT1 in the parietal cortex of AD patients compared with the control, and the accumulation of $\mathrm{A} \beta$ and tau in the AD patients may be associated with the loss of SirT1 [29]. Julien et al. suggested that the mutant mice with the lack of SirT1 show impairment in memory and synaptic plasticity, and SirT1 modulates synaptic plasticity and memory formation partly via the way of upregulation of miR-134 [30]. Therefore, SirT1 could be a target for the treatment of neurodegenerative disorders. Additionally, overexpression of the $\mathrm{NAD}^{+}$-dependent deacetylase SirT1 in a mouse model of $\mathrm{AD}$ reduces the production of $\mathrm{A} \beta$ and formation of plaques through activating the transcription of gene encoding the $\alpha$ secretase ADAM10. SirT1 also regulates the Notch pathway, which repairs neuronal damage in brains [31].

The level of $\alpha$-tubulin acetylation is known to play an important role in microtubule stability and SirT2 decreases the level of $\alpha$-tubulin deacetylation [14]. Taylor et al. suggested that inhibition activity of SirT2 deacetylase reduces total cholesterol in primary striatal neurons [32]. The above facts hint that Sir2 is involved in the pathology of AD.

Kawamura et al. found out that RNAi-mediated SirT3 knockdown increases mitochondrial reactive oxygen species 


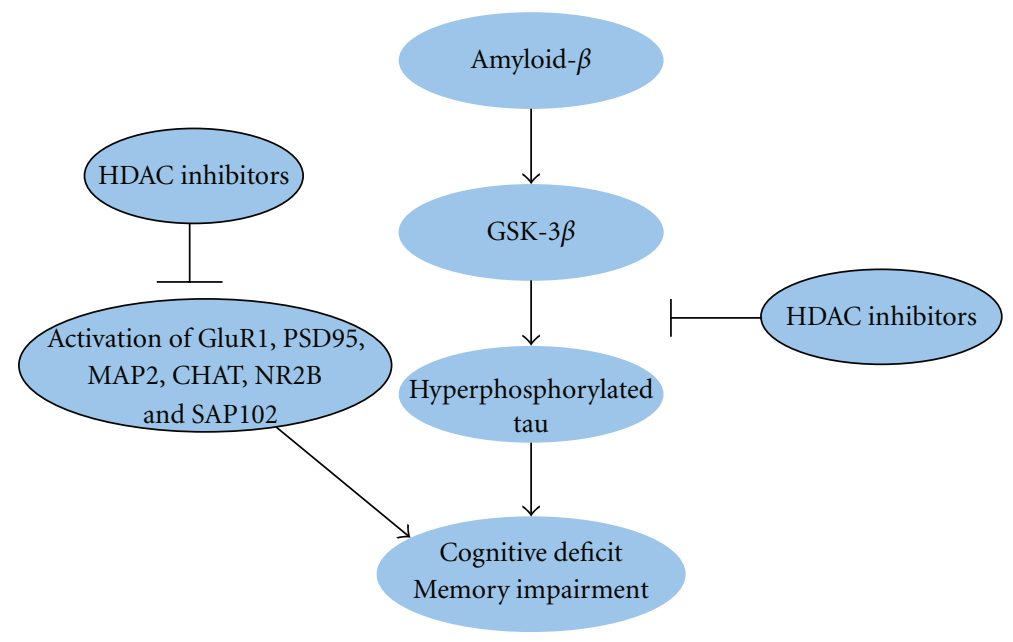

FIgure 1: The role of HDAC inhibitors in Alzheimer's disease. First, HDAC inhibitors inhibit A $\beta$-induced hyperphosphorylation of tau protein. Second, HDAC inhibitors alter the expression of important genes which participate in the learning and memory.

(ROS) generation in mouse fertilized eggs, and the mitochondrial ROS generation is accompanied by the p53 upregulation in SirT3-knockdown mouse embryos [33]. Additionally, the primary cultured mouse cortical neurons treated with NMDA induce massive ROS production as well as the increase of mitochondrial SirT3, while overexpression of SirT3 significantly reduces the mitochondrial ROS generation [34]. Therefore, the SirT3 appears to play a role in the protection of the nervous system against excitotoxicity.

\section{Summaries}

$\mathrm{AD}$ is one of the most common forms of dementia. Currently, the pathology of this disease is not fully understood. "Amyloid cascade hypothesis" states that the increased $\mathrm{A} \beta$ causes the disease progress, such as cognitive deficits, memory impairment, and neuron loss. Accumulating evidence supports the view that the HDAC proteins may be involved in its development. The HDAC proteins may regulate the level of histone acetylation and then alter the expression of some important genes which are involved in the memory and cognition and pathology of AD. HDAC inhibitors may ameliorate cognitive deficits and memory impairment in $\mathrm{AD}$ animal models. The potential pathways of HDAC inhibitors reversing $\mathrm{A} \beta$-induced neurotoxicity may lie in that (1) HDAC inhibitors inhibit $\mathrm{A} \beta$-induced hyperphosphorylation of tau protein; (2) HDAC inhibitors may regulate the expression of important genes that participate in the learning and memory (Figure 1). However, many issues need to be resolved before these inhibitors can be used to treat AD. For example, it is not yet clear which subtypes of HDACs are associated with the AD and which selective HDAC inhibitors would be effective to treat AD. Further researches are needed to clarify the exact role of HDAC proteins and to develop their selective inhibitors in the pathology of AD.

\section{Acknowledgments}

This research was supported by Project 31071512 supported by the National Natural Science Foundation of China and by the Funding Project for Academic Human Resources Development in Institutions of Higher Learning under the Jurisdiction of Beijing Municipality (PHR(IHLB)) (PHR20090514).

\section{References}

[1] J. Hardy, "A hundred years of Alzheimer's disease research," Neuron, vol. 52, no. 1, pp. 3-13, 2006.

[2] J. Hardy and D. J. Selkoe, "The amyloid hypothesis of Alzheimer's disease: progress and problems on the road to therapeutics," Science, vol. 297, no. 5580, pp. 353-356, 2002.

[3] J. A. Hardy and G. A. Higgins, "Alzheimer's disease: the amyloid cascade hypothesis," Science, vol. 256, no. 5054, pp. 184-185, 1992.

[4] L. M. Bekris, C. E. Yu, T. D. Bird, and D. W. Tsuang, "Genetics of Alzheimer disease," Journal of Geriatric Psychiatry and Neurology, vol. 23, no. 4, pp. 213-227, 2010.

[5] E. Korzus, "Manipulating the brain with epigenetics," Nature Neuroscience, vol. 13, no. 4, pp. 405-406, 2010.

[6] R. M. Stilling and A. Fischer, "The role of histone acetylation in age-associated memory impairment and Alzheimer's disease," Neurobiology of Learning and Memory, vol. 96, no. 1, pp. 19-26, 2011.

[7] S. E. Johnstone and S. B. Baylin, "Stress and the epigenetic landscape: a link to the pathobiology of human diseases?" Nature Reviews Genetics, vol. 11, no. 11, pp. 806-812, 2010.

[8] S. Y. Roth, J. M. Denu, and C. D. Allis, "Histone acetyltransferases," Annual Review of Biochemistry, vol. 70, pp. 81-120, 2001.

[9] D. M. Chuang, Y. Leng, Z. Marinova, H. J. Kim, and C. T. Chiu, "Multiple roles of HDAC inhibition in neurodegenerative conditions," Trends in Neurosciences, vol. 32, no. 11, pp. 591$601,2009$.

[10] N. Carey and N. B. la Thangue, "Histone deacetylase inhibitors: gathering pace," Current Opinion in Pharmacology, vol. 6, no. 4, pp. 369-375, 2006. 
[11] E. Michishita, J. Y. Park, J. M. Burneskis, J. C. Barrett, and I. Horikawa, "Evolutionarily conserved and nonconserved cellular localizations and functions of human SIRT proteins," Molecular Biology of the Cell, vol. 16, no. 10, pp. 4623-4635, 2005.

[12] A. J. de Ruijter, A. H. van Gennip, H. N. Caron, S. Kemp, and A. B. P. van Kuilenburg, "Histone deacetylases (HDACs): characterization of the classical HDAC family," Biochemical Journal, vol. 370, no. 3, pp. 737-749, 2003.

[13] L. Gao, M. A. Cueto, F. Asselbergs, and P. Atadja, "Cloning and functional characterization of HDAC11, a novel member of the human histone deacetylase family," Journal of Biological Chemistry, vol. 277, no. 28, pp. 25748-25755, 2002.

[14] K. N. Green, J. S. Steffan, H. Martinez-Coria et al., "Nicotinamide restores cognition in Alzheimer's disease transgenic mice via a mechanism involving sirtuin inhibition and selective reduction of Thr231-phosphotau," Journal of Neuroscience, vol. 28, no. 45, pp. 11500-11510, 2008.

[15] Y. Su, J. Ryder, B. Li et al., "Lithium, a common drug for bipolar disorder treatment, regulates amyloid- $\beta$ precursor protein processing," Biochemistry, vol. 43, no. 22, pp. 6899-6908, 2004.

[16] H. Qing, G. He, P. T. T. Ly et al., "Valproic acid inhibits a $\beta$ production, neuritic plaque formation, and behavioral deficits in alzheimer's disease mouse models," Journal of Experimental Medicine, vol. 205, no. 12, pp. 2781-2789, 2008.

[17] A. Ricobaraza, M. C. Tejedor, A. P. Mediavilla, D. Frechilla, J. del Río, and A. G. Osta, "Phenylbutyrate ameliorates cognitive deficit and reduces $\tau$ pathology in an alzheimer's disease mouse model," Neuropsychopharmacology, vol. 34, no. 7, pp. 1721-1732, 2009.

[18] A. Ricobaraza, "Phenylbutyrate rescues dendriticspine loss associated with memory deficits in a mouse model of Alzheimer disease," Hippocampus. In press.

[19] H. Ding, P. J. Dolan, and G. V. Johnson, "Histone deacetylase 6 interacts with the microtubule-associated protein $\tau$," Journal of Neurochemistry, vol. 106, no. 5, pp. 2119-2130, 2008.

[20] O. Bruserud, C. Stapnes, E. Ersvær, B. T. Gjertsen, and A. Ryningen, "Histone deacetylase inhibitors in cancer treatment: a review of the clinical toxicity and the modulation of gene expression in cancer cell," Current Pharmaceutical Biotechnology, vol. 8, no. 6, pp. 388-400, 2007.

[21] N. M. Tsankova, O. Berton, W. Renthal, A. Kumar, R. L. Neve, and E. J. Nestler, "Sustained hippocampal chromatin regulation in a mouse model of depression and antidepressant action," Nature Neuroscience, vol. 9, no. 4, pp. 519-525, 2006.

[22] S. J. Haggarty, K. M. Koeller, J. C. Wong, C. M. Grozinger, and S. L. Schreiber, "Domain-selective small-molecule inhibitor of histone deacetylase 6 (HDAC6)-mediated tubulin deacetylation," Proceedings of the National Academy of Sciences of the United States of America, vol. 100, no. 8, pp. 4389-4394, 2003.

[23] J. Trapp, R. Meier, D. Hongwiset, M. U. Kassack, W. Sippl, and M. Jung, "Structure-activity studies on suramin analogues as inhibitors of $\mathrm{NAD}^{+}$-dependent histone deacetylases (sirtuins)," ChemMedChem, vol. 2, no. 10, pp. 1419-1431, 2007.

[24] S. Peleg, F. Sananbenesi, A. Zovoilis et al., "Altered histone acetylation is associated with age-dependent memory impairment in mice," Science, vol. 328, no. 5979, pp. 753-756, 2010.

[25] M. Kilgore, C. A. Miller, D. M. Fass et al., "Inhibitors of class 1 histone deacetylases reverse contextual memory deficits in a mouse model of alzheimer's disease," Neuropsychopharmacology, vol. 35, no. 4, pp. 870-880, 2010.

[26] Y. I. Francis, M. Fà, H. Ashraf et al., "Dysregulation of histone acetylation in the APP/PS1 mouse model of Alzheimer's disease," Journal of Alzheimer's Disease, vol. 18, no. 1, pp. 131139, 2009.
[27] Z. Zhang, R. Zhao, J. Qi, S. Wen, Y. Tang, and D. Wang, "Inhibition of glycogen synthase kinase- $3 \beta$ by Angelica sinensis extract decreases $\beta$-amyloid-induced neurotoxicity and $\tau$ phosphorylation in cultured cortical neurons," Journal of Neuroscience Research, vol. 89, no. 3, pp. 437-447, 2011.

[28] J. Huang, Y. J. Chen, W. H. Bian, J. Yu, Y. W. Zhao, and X. Y. Liu, "Unilateral amyloid- $\beta 25-35$ injection into the rat amygdala increases the expressions of aberrant $\tau$ phosphorylation kinases," Chinese Medical Journal, vol. 123, no. 10, pp. 1311-1314, 2010.

[29] J. Gao, W. Y. Wang, Y. W. Mao et al., "A novel pathway regulates memory and plasticity via SIRT1 and miR-134," Nature, vol. 466, no. 7310, pp. 1105-1109, 2010.

[30] C. Julien, C. Tremblay, V. Émond et al., "Sirtuin 1 reduction parallels the accumulation of $\tau$ in alzheimer disease," Journal of Neuropathology and Experimental Neurology, vol. 68, no. 1, pp. 48-58, 2009.

[31] G. Donmez, D. Wang, D. E. Cohen, and L. Guarente, "SIRT1 suppresses $\beta$-amyloid production by activating the $\alpha$-secretase gene ADAM10," Cell, vol. 142, no. 2, pp. 320-332, 2010.

[32] D. M. Taylor, U. Balabadra, Z. Xiang et al., "A brain-permeable small molecule reduces neuronal cholesterol by inhibiting activity of sirtuin 2 deacetylase," ACS Chemical Biology, vol. 6, no. 6, pp. 540-546, 2011.

[33] Y. Kawamura, Y. Uchijima, N. Horike et al., "Sirt3 protects in vitro-fertilized mouse preimplantation embryos against oxidative stress-Induced p53-mediated developmental arrest," Journal of Clinical Investigation, vol. 120, no. 8, pp. 2817-2828, 2010.

[34] S. H. Kim, H. F. Lu, and C. C. Alano, "Neuronal sirt3 protects against excitotoxic injury in mouse cortical neuron culture," PLoS ONE, vol. 6, no. 3, 2011.

[35] J. S. Guan, S. J. Haggarty, E. Giacometti et al., "HDAC2 negatively regulates memory formation and synaptic plasticity," Nature, vol. 459, no. 7243, pp. 55-60, 2009.

[36] M. W. Akhtar, J. Raingo, E. D. Nelson et al., "Histone deacetylases 1 and 2 form a developmental switch that controls excitatory synapse maturation and function," Journal of Neuroscience, vol. 29, no. 25, pp. 8288-8297, 2009.

[37] S. C. McQuown, R. M. Barrett, D. P. Matheos et al., "HDAC3 is a critical negative regulator of long-term memory formation," Journal of Neuroscience, vol. 31, no. 2, pp. 764-774, 2011.

[38] F. H. Bardai and S. R. d'Mello, "Selective toxicity by HDAC3 in neurons: regulation by Akt and GSK3 $\beta$," Journal of Neuroscience, vol. 31, no. 5, pp. 1746-1751, 2011.

[39] Z. Zhao, H. Xu, and W. Gong, "Histone deacetylase 6 (HDAC6) is an independent deacetylase for $\alpha$-tubulin," Protein and Peptide Letters, vol. 17, no. 5, pp. 555-558, 2010.

[40] P. Bali, M. Pranpat, J. Bradner et al., "Inhibition of histone deacetylase 6 acetylates and disrupts the chaperone function of heat shock protein 90: a novel basis for antileukemia activity of histone deacetylase inhibitors," Journal of Biological Chemistry, vol. 280, no. 29, pp. 26729-26734, 2005.

[41] S. Chen, G. C. Owens, H. Makarenkova, and D. B. Edelman, "HDAC6 regulates mitochondrial transport in hippocampal neurons," PloS one, vol. 5, no. 5, 2010.

[42] M. A. Rivieccio, C. Brochier, D. E. Willis et al., "HDAC6 is a target for protection and regeneration following injury in the nervous system," Proceedings of the National Academy of Sciences of the United States of America, vol. 106, no. 46, pp. 19599-19604, 2009.

[43] T. A. Bolger and T. P. Yao, "Intracellular trafficking of histone deacetylase 4 regulates neuronal cell death," Journal of Neuroscience, vol. 25, no. 41, pp. 9544-9553, 2005. 


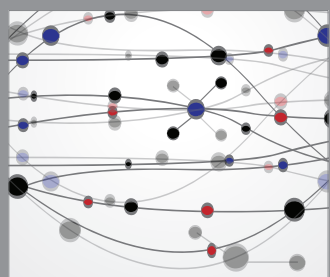

The Scientific World Journal
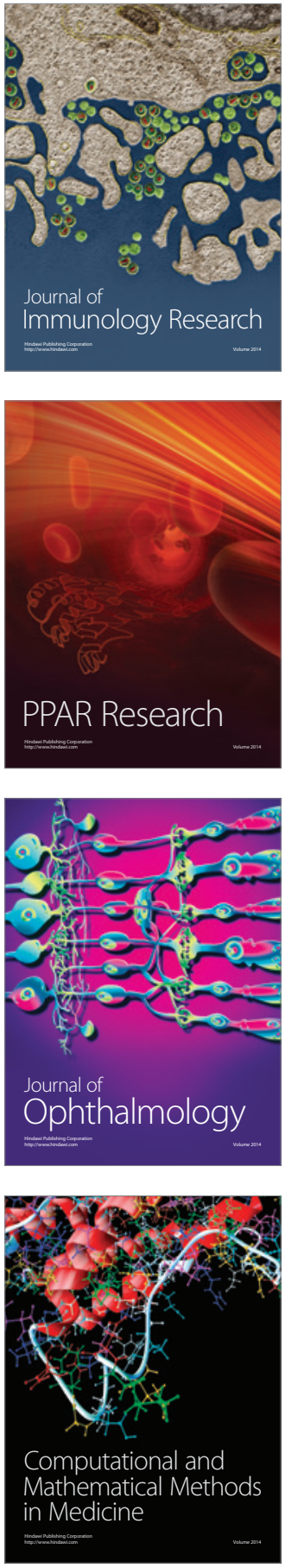

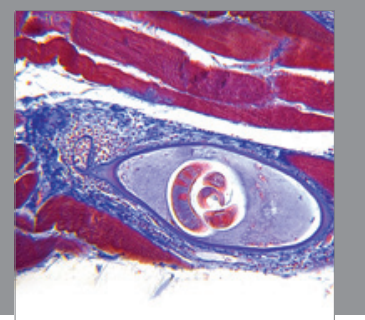

Gastroenterology

Research and Practice
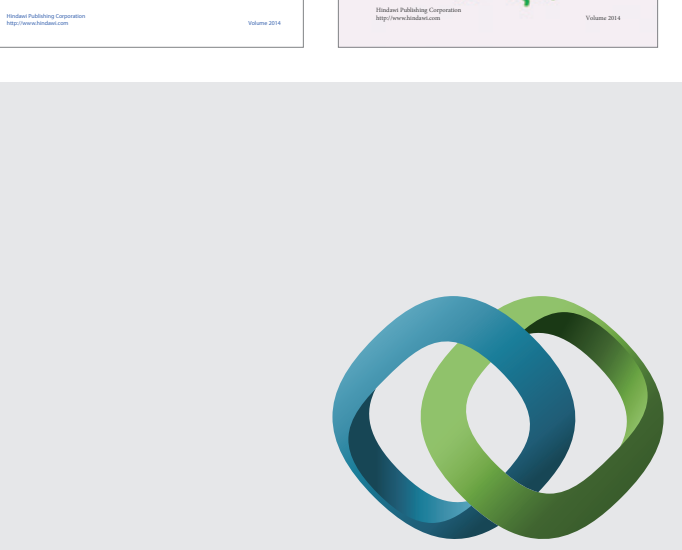

\section{Hindawi}

Submit your manuscripts at

http://www.hindawi.com
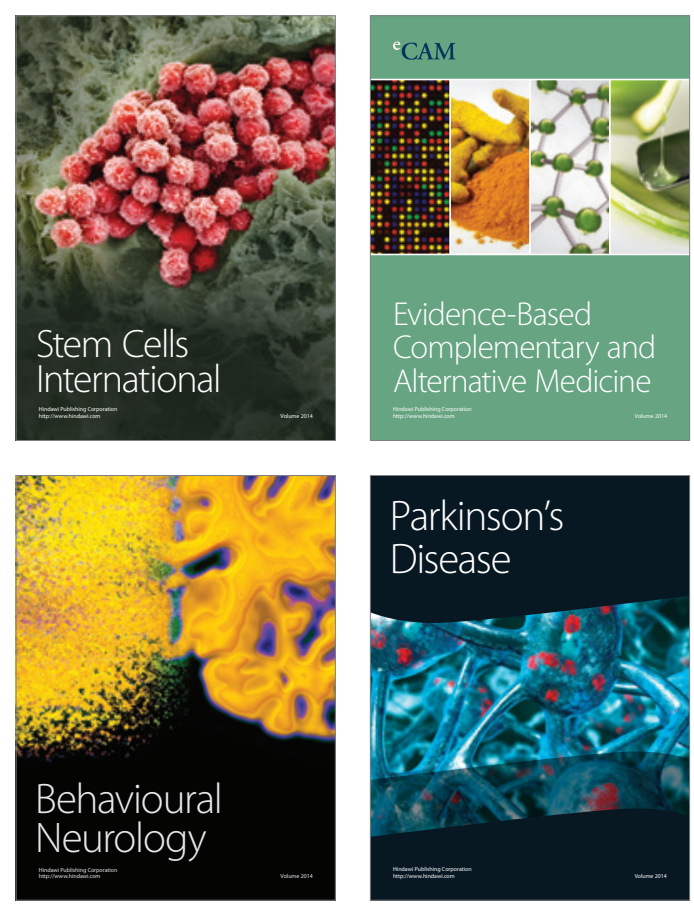

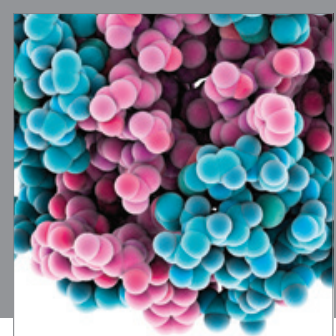

Journal of
Diabetes Research

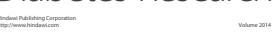

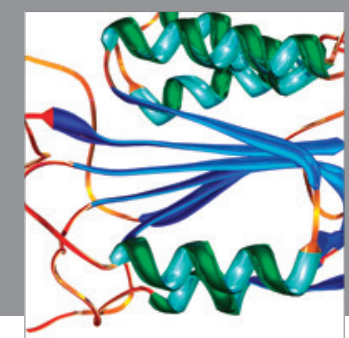

Disease Markers
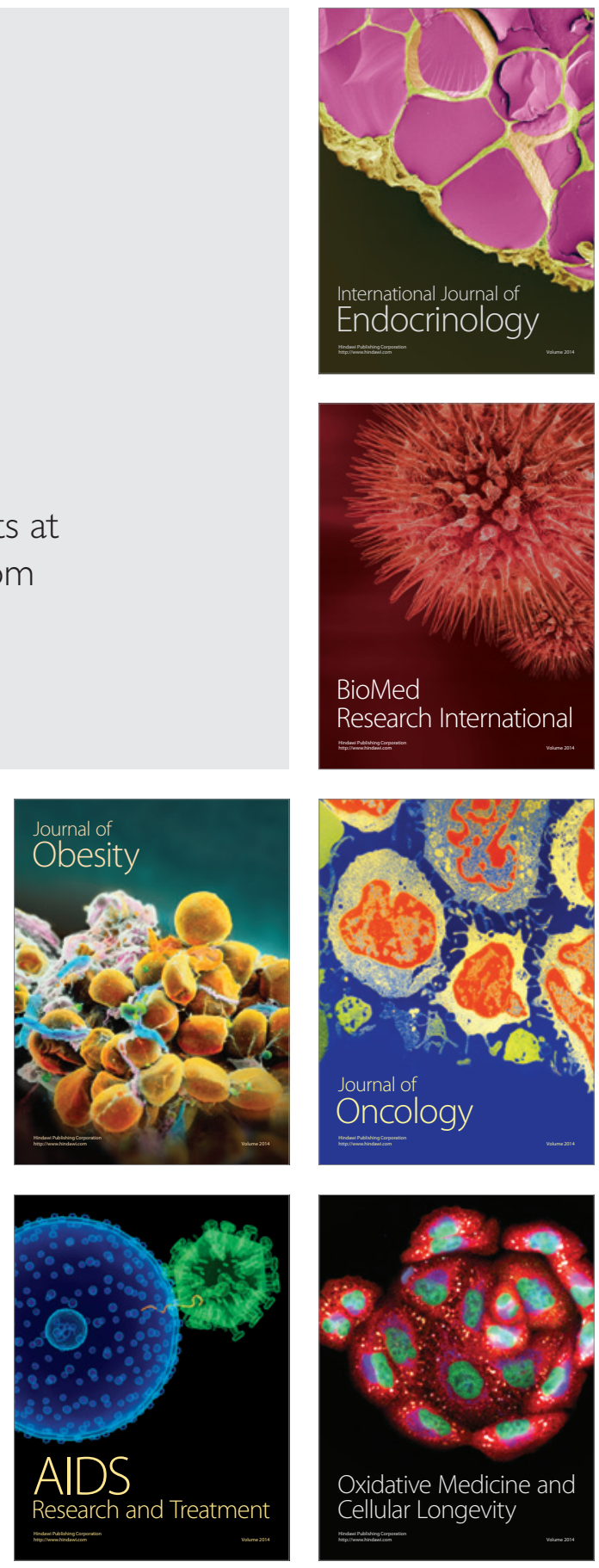